PROCEEDINGS OF THE

AMERICAN MATHEMATICAL SOCIETY

Volume 130, Number 2, Pages 555-563

S 0002-9939(01)06060-9

Article electronically published on June 19, 2001

\title{
AN ELEMENTARY PROOF OF SHARP SOBOLEV EMBEDDINGS
}

\author{
JAN MALÝ AND LUBOŠ PICK
}

(Communicated by Joseph A. Ball)

\begin{abstract}
We present an elementary unified and self-contained proof of sharp Sobolev embedding theorems. We introduce a new function space and use it to improve the limiting Sobolev embedding theorem due to Brézis and Wainger.
\end{abstract}

\section{Prologue}

Let $\Omega$ be an open subset of $\mathbb{R}^{n}$, where $n \geq 2$, let $1 \leq p<\infty$ and let $W^{1, p}(\Omega)$ be the Sobolev space, that is, the set of all functions in $L^{p}(\Omega)$, whose distributional derivatives of the first order belong to $L^{p}(\Omega)$, too. If $p=n$ we assume that $|\Omega|<\infty$. We define $W_{0}^{1, p}(\Omega)$ as the closure of $C_{0}^{\infty}(\Omega)$ in $W^{1, p}(\Omega)$.

We denote

$$
p^{*}=\frac{n p}{n-p}, \quad 1 \leq p<n
$$

The classical Sobolev theorem [16] asserts that

$$
W_{0}^{1, p}(\Omega) \hookrightarrow L^{p^{*}}(\Omega) \quad \text { when } 1<p<n .
$$

(As usual, $\hookrightarrow$ stands for a continuous embedding.) Although $p^{*}$ tends to infinity as $p \rightarrow n-$, the space $W_{0}^{1, n}(\Omega)$ contains unbounded functions. Instead of an embedding into $L^{\infty}(\Omega)$, one has

$$
W_{0}^{1, n}(\Omega) \hookrightarrow \exp L^{\frac{n}{n-1}}(\Omega),
$$

where $\exp L^{\frac{n}{n-1}}(\Omega)$ is the exponential-type Orlicz space endowed with the norm

$$
\|u\|_{\exp L^{\frac{n}{n-1}(\Omega)}}=\inf \left\{\lambda>0 ; \int_{\Omega} \exp \left(\left(\frac{|u(x)|}{\lambda}\right)^{\frac{n}{n-1}}\right) d x \leq 1\right\} .
$$

The embedding (1.2) is usually associated to Trudinger [19]; similar results had been obtained earlier by Pokhozhaev [15] and Yudovich [20].

Now, both of the embeddings (1.1) and (1.2) are sharp within the context of Orlicz spaces. In other words, neither of the target spaces can be replaced by an essentially smaller Orlicz space. This fact was observed by Hempel, Morris and Trudinger [9] for (1.2); a general result was later obtained by Cianchi 5]. However,

Received by the editors May 3, 2000 and, in revised form, July 14, 2000

1991 Mathematics Subject Classification. Primary 46E35; Secondary 46E30, 26D10.

(C)2001 American Mathematical Society 
both of the target spaces can be improved if we are willing to allow different function spaces than Orlicz spaces. Consider Lorentz spaces $L^{p, q}(\Omega)$, defined by

$$
L^{p, q}(\Omega):=\left\{u \text { measurable on } \Omega ;\|u\|_{L^{p, q}(\Omega)}=\left\|t^{\frac{1}{p}-\frac{1}{q}} u^{*}(t)\right\|_{L^{q}(0, \infty)}<\infty\right\},
$$

where $p, q \in(0, \infty]$ and

$$
u^{*}(t)=\inf \{\lambda>0 ;|\{x \in \Omega ;|u(x)|>\lambda\}| \leq t\}, \quad t \in(0, \infty)
$$

is the non-increasing rearrangement of $u$. Then (1.1) can be replaced by

$$
W_{0}^{1, p}(\Omega) \hookrightarrow L^{p^{*}, p}(\Omega) \quad \text { when } 1 \leq p<n,
$$

which is a sharper inclusion than (1.1), since $L^{p^{*}, p}(\Omega) \varsubsetneqq L^{p^{*}}(\Omega)$. This was observed by O'Neil [13] and Peetre [14]. An analogous improvement of (1.2) is possible, too, but Lorentz spaces no longer suffice to do the job; we need a more complicated function space. Namely, we have

$$
W_{0}^{1, n}(\Omega) \hookrightarrow B W_{n}(\Omega)
$$

where

$$
\|u\|_{B W_{p}(\Omega)}=\left(\int_{0}^{|\Omega|}\left(\frac{u^{*}(t)}{\log \left(\frac{|\Omega|}{t}\right)}\right)^{p} \frac{d t}{t}\right)^{\frac{1}{p}} \quad \text { for } 0<p<\infty .
$$

Again, (1.4) is a sharper result than (1.2) as $B W_{n}(\Omega) \varsubsetneqq \exp L^{\frac{n}{n-1}}(\Omega)$. The embedding (1.4) is due to Brézis and Wainger [4] and independently to Hansson [8]. It can also be derived from capacitary estimates of Maz'ya [12]. It follows from [6] Theorem 5.11] that both (1.3) and (1.4) are sharp within the context of rearrangement-invariant Banach function spaces. That is, neither the space $L^{p^{*}, p}(\Omega)$ in (1.3) nor $B W_{n}(\Omega)$ in (1.4) can be replaced by any essentially smaller rearrangementinvariant Banach function space.

The aim of this paper is two-fold. First, we wish to contribute to the discussion on how to teach (sharp) Sobolev embeddings in advanced courses. We have compiled an elementary unified proof, which we are going to show in a self-contained way. We first establish the weak version of the Sobolev-Gagliardo-Nirenberg embedding, that is,

$$
\lambda(|\{|u| \geq \lambda\}|)^{\frac{1}{n^{\prime}}} \leq C \int_{\Omega}|\nabla u| d x
$$

for all $u \in W_{0}^{1,1}(\Omega)$ and $\lambda>0$. In our opinion, it is not reasonable to avoid potential estimates in lecture courses on function spaces. It is natural to make profit of this knowledge when deriving (1.5). This approach seems to be less tricky than the traditional way of proving strong-type Gagliardo-Nirenberg estimates. Now, having (1.5) at our disposal, the remaining job, namely the proof of (1.3) and (1.4), can be made very simple and elementary, and it is not essentially more difficult to obtain sharp embeddings than the classical $L^{q}$-ones.

For the sake of simplicity we restrict ourselves to the space $W_{0}^{1, p}(\Omega)$. If the domain verifies additional boundedness and regularity assumptions, then the argument can be easily modified to obtain embedding theorems for $W^{1, p}(\Omega)$ or Poincaré type inequalities. 
Now when we know that (1.3) and (1.4) are sharp in the context of rearrangement-invariant Banach function spaces, we can rewrite them in the form of inequalities. For their formulation and proof, we can even forget the notion of a Lorentz space.

Theorem A. Assume that $1 \leq p<n$. Then

$$
\int_{0}^{|\Omega|} t^{\frac{p}{p^{*}}-1} u^{*}(t)^{p} d t \leq C \int_{\Omega}|\nabla u(x)|^{p} d x
$$

for all $u \in W_{0}^{1, p}(\Omega)$.

Theorem B. Assume that $|\Omega|<\infty$. Then

$$
\int_{0}^{|\Omega|}\left(\frac{u^{*}(t)}{\log \left(\frac{|\Omega|}{t}\right)}\right)^{n} \frac{d t}{t} \leq C \int_{\Omega}|\nabla u|^{n}(x) d x
$$

for all $u \in W_{0}^{1, p}(\Omega)$.

Theorems A and B are proved in Section 2, Concerning the proofs, we do not claim that our approach is original. A lot of work has been done and our aim is to optimize the argument. In main features we follow the strategy used by Tartar in his recent paper 18, where the inequalities (1.3) and (1.4) (and many others) are also proved. Concerning details, it would be very hard to trace back sources of each particular trick.

The fact that strong-type Sobolev estimates can be derived from weak ones has been observed by Federer 7 when deriving the Sobolev-Gagliardo-Nirenberg inequality $(p=1)$ from the isoperimetric inequality. The co-area integral argument can be simplified by the truncation trick which has been invented by Maz'ya [11] in connection with capacitary estimates. Its application to getting strong-type Sobolev embeddings from their weak forms is pursued for example by Bakry, Coulhon, Ledoux and Saloff-Coste in [1, where plenty of references are also given. The point of departure in [1] is the well-known equivalence of the Sobolev embedding to Nash and Moser inequalities, which is avoided here.

The second goal consists of the introduction and examination of a new function space. In the course of the proof of Theorem B we discover that when $|\nabla u|^{n}$ is integrable over $\Omega$, then $u$ in fact belongs to a yet smaller class than $B W_{n}(\Omega)$. We denote this class by $W_{n}(\Omega)$. In general, we define $W_{p}(\Omega)$ for $\Omega$ bounded and $1 \leq p<\infty$ as the family of all measurable functions on $\Omega$ for which

$$
\|u\|_{W_{p}(\Omega)}:=\left(\int_{0}^{|\Omega|} \frac{\left(u^{*}\left(\frac{t}{2}\right)-u^{*}(t)\right)^{p}}{t} d t\right)^{\frac{1}{p}}<\infty .
$$

Note that $u \in W_{p}(\Omega)$ if and only if

$$
\sum_{k=1}^{\infty}\left(u^{*}\left(2^{-k}|\Omega|\right)-u^{*}\left(2^{1-k}|\Omega|\right)\right)^{p}<\infty .
$$

In the last section we study the properties of $W_{p}(\Omega)$; perhaps the most interesting one is the fact that it is not closed with respect to addition of functions (in particular, it is not a linear set). In view of this, $W_{p}(\Omega)$ somewhat resembles the space weak $-L^{\infty}$ of Bennett, De Vore and Sharpley [2]. 
The space $W_{p}(\Omega)$ is interesting from two points of view: first, it is a qualitatively new class of functions which might find its applications in various parts of analysis, and, second, it enables us to obtain a non-trivial improvement of the Sobolev embedding in a limiting case, the sharpest known thus far. At this moment we are not aware of any further connections of this space to practical tasks, but it is quite possible that it might play a role in interpolation theory, analogous to the one played by weak- $L^{\infty}$ (cf. [3, Chapter 5 , Section 7]).

Throughout the paper, $C$ stands for a positive constant, not necessarily the same at each occurrence.

\section{Elementary proof of EMBeddings}

We proceed in two steps. First we sketch an elementary proof of (1.5) and then show that it implies both (1.6) and (1.7).

Lemma 2.1. For every $u \in W_{0}^{1,1}(\Omega)$ and $\lambda>0$, the estimate (1.5) holds.

Proof. For $x \in \mathbb{R}^{n}$ and $R>0$, denote $B(x, R)=\left\{y \in \mathbb{R}^{n} ;|y|<R\right\}$, and let $\omega_{n}$ be the volume of the $n$-dimensional unit ball. Then an elementary calculation shows that

$$
\int_{B(0, R)}|x|^{1-n} d x=\omega_{n} R
$$

We claim that for every $y \in \mathbb{R}^{n}$ and $G \subset \mathbb{R}^{n}$ of positive finite measure

$$
\int_{G}|x-y|^{1-n} d x \leq \omega_{n}^{1-\frac{1}{n}}|G|^{\frac{1}{n}} .
$$

Indeed, fix such $G$ and $y$ and let $B=B(y, R)$ be such that $|B|=|G|$. Then also $|B \backslash G|=|G \backslash B|$, hence

$$
\int_{G \backslash B}|x-y|^{1-n} d x \leq \int_{G \backslash B} R^{1-n}=\int_{B \backslash G} R^{1-n} \leq \int_{B \backslash G}|x-y|^{1-n} d x .
$$

Thus, adding $\int_{G \cap B}$ to both sides, we get by 2.1.

$$
\int_{G}|x-y|^{1-n} d x \leq \int_{B}|x-y|^{1-n} d x \leq \omega_{n} R=\omega_{n}^{1-\frac{1}{n}}|B|^{\frac{1}{n}}=\omega_{n}^{1-\frac{1}{n}}|G|^{\frac{1}{n}} .
$$

Now, fix $u \in C_{0}^{\infty}(\Omega)$. Extended by zero outside $\Omega$, we can consider $u \in C_{0}^{\infty}\left(\mathbb{R}^{n}\right)$. We denote by $G$ the set $\left\{x \in \mathbb{R}^{n} ;|u(x)| \geq \lambda\right\}$. Let $K \subset G$ be a compact set. Then, by the well-known integral representation (cf. e.g. [17, Chapter 5, (18), p. 125]), Fubini's theorem and (2.2),

$$
\begin{aligned}
\lambda|K| & \leq \int_{K}|u(x)| d x \leq C \int_{K} \int_{\mathbb{R}^{n}} \frac{|\nabla u(y)|}{|x-y|^{n-1}} d y d x \\
& \leq C \int_{\mathbb{R}^{n}}|\nabla u(y)| \int_{K} \frac{d x}{|x-y|^{n-1}} d y \leq C|K|^{\frac{1}{n}} \int_{\mathbb{R}^{n}}|\nabla u(y)| d y .
\end{aligned}
$$

Thus, since $|K|<\infty$,

$$
\lambda|K|^{1-\frac{1}{n}} \leq C \int_{\mathbb{R}^{n}}|\nabla u(y)| d y,
$$

and in turn, on letting $K \uparrow G$,

$$
\lambda|G|^{1-\frac{1}{n}} \leq C \int_{\mathbb{R}^{n}}|\nabla u(y)| d y .
$$


This shows (1.5) for $u \in C_{0}^{\infty}(\Omega)$. The general case follows by a standard approximation argument.

Remark 2.2. The weak estimate (1.5) is usually proved by means of HardyLittlewood maximal inequalities based on covering techniques. Our proof avoids the use of covering tricks. Although each step is a rather well-known routine, we were not able to find such a proof in its entirety in the literature. Another elementary proof based on a simple interpolation can be found in the book [17] by Stein.

Lemma 2.3. Let $|\Omega|<\infty$ and $1 \leq p \leq n$. Let $u \in W_{0}^{1, p}(\Omega)$ and denote

$$
t_{k}=2^{1-k}|\Omega| \quad \text { and } \quad a_{k}=u^{*}\left(t_{k}\right), \quad k \in \mathbb{N} .
$$

Then

$$
\sum_{k=1}^{\infty} t_{k}^{\frac{p}{p^{*}}}\left(a_{k+1}-a_{k}\right)^{p} \leq C \int_{\Omega}|\nabla u|^{p} d x
$$

with $C$ depending only on $p$ and $n$.

Proof. If $p>1$, using the Hölder inequality at the right-hand side of (1.5) we obtain

$$
\lambda(|\{|u| \geq \lambda\}|)^{\frac{1}{n^{\prime}}} \leq C\left(\int_{\Omega}|\nabla u|^{p} d x\right)^{\frac{1}{p}}(|\{|u|>0\}|)^{\frac{1}{p^{\prime}}}, \quad \lambda \in(0, \infty) .
$$

With the convention $\frac{1}{1^{\prime}}=0$ this also holds for $p=1$. Now, given $0<a<b<\infty$, we use a smooth function $\varphi_{a}^{b}$ on $\mathbb{R}$ such that

$$
\begin{cases}\varphi_{a}^{b}(s)=0 & \text { for } s \in(-\infty, a], \\ 0<\left(\varphi_{a}^{b}\right)^{\prime}(s)<2 & \text { for } s \in(a, b), \\ \varphi_{a}^{b}(s)=b-a & \text { for } s \in[b, \infty) .\end{cases}
$$

Applying 2.5 to the function $\varphi_{a}^{b} \circ|u|$ and $\lambda=(b-a)$, we arrive at

$$
(b-a)(|\{|u| \geq b\}|)^{\frac{1}{n^{\prime}}} \leq C\left(\int_{a<|u|<b}|\nabla u|^{p} d x\right)^{\frac{1}{p}}(|\{|u|>a\}|)^{\frac{1}{p^{\prime}}} .
$$

Then

$$
\left|\left\{|u|>a_{k}\right\}\right| \leq t_{k} \leq\left|\left\{|u| \geq a_{k}\right\}\right|,
$$

and thus (2.6) applied to $a=a_{k}$ and $b=a_{k+1}$ yields

$$
t_{k+1}^{\frac{1}{n^{\prime}}}\left(a_{k+1}-a_{k}\right) \leq C\left(\int_{a_{k}<u<a_{k+1}}|\nabla u|^{p} d x\right)^{\frac{1}{p}} t_{k}^{\frac{1}{p^{\prime}}},
$$

that is (recall that $2 t_{k+1}=t_{k}$ ),

$$
t_{k}^{\frac{1}{p^{*}}}\left(a_{k+1}-a_{k}\right) \leq C\left(\int_{a_{k}<u<a_{k+1}}|\nabla u|^{p} d x\right)^{\frac{1}{p}},
$$

where the convention $\frac{1}{n^{*}}=0$ is used. We raise this estimate to the power $p$ and sum over $k$. We obtain

$$
\sum_{k=1}^{\infty} t_{k}^{\frac{p}{p^{*}}}\left(a_{k+1}-a_{k}\right)^{p} \leq C \sum_{k=1}^{\infty} \int_{a_{k}<u<a_{k+1}}|\nabla u|^{p} d x \leq C \int_{\Omega}|\nabla u|^{p} d x,
$$

finishing the proof. 
Corollary 2.4. If $|\Omega|<\infty$, then

$$
W_{0}^{1, n}(\Omega) \hookrightarrow W_{n}(\Omega) .
$$

Proof. Note that, for $p=n,(2.4)$ reads as

$$
\sum_{k=1}^{\infty}\left(a_{k+1}-a_{k}\right)^{n} \leq C \int_{\Omega}|\nabla u|^{n} d x
$$

which is just a discrete version of (2.7).

Proof of Theorem A. Let us first assume that $|\Omega|<\infty$ and fix $u \in W_{0}^{1, p}(\Omega) \cap$ $L^{\infty}(\Omega)$. Let $t_{k}$ and $a_{k}$ have the same meaning as in (2.3). Given $\varepsilon>0$, the convexity of $t^{p}$ yields ([10, Lemma 1.1])

$$
a_{k+1}^{p} \leq\left(1+\frac{1}{\varepsilon}\right)^{p-1}\left(a_{k+1}-a_{k}\right)^{p}+(1+\varepsilon)^{p-1} a_{k}^{p} .
$$

Hence (taking into account that $a_{1}=u^{*}\left(t_{1}\right)=0$ )

$$
\begin{aligned}
& 2^{\frac{p}{p^{*}}} \sum_{k=1}^{\infty} t_{k+1}^{\frac{p}{p^{*}}} a_{k}^{p}=\sum_{k=1}^{\infty} t_{k+1}^{\frac{p}{p^{*}}} a_{k+1}^{p} \\
& \quad \leq(1+\varepsilon)^{p-1} \sum_{k=1}^{\infty} t_{k+1}^{\frac{p}{p^{*}}} a_{k}^{p}+\left(1+\frac{1}{\varepsilon}\right)^{p-1} \sum_{k=1}^{\infty} t_{k+1}^{\frac{p}{p^{*}}}\left(a_{k+1}-a_{k}\right)^{p} .
\end{aligned}
$$

Choosing $\varepsilon>0$ so small that

$$
(1+\varepsilon)^{p-1}<2^{\frac{p}{p^{*}}}
$$

we obtain

$$
\sum_{k=1}^{\infty} t_{k+1}^{\frac{p}{p^{*}}}\left(u^{*}\left(t_{k}\right)\right)^{p} \leq C \int_{\Omega}|\nabla u|^{p} d x
$$

which is a discrete version of (1.6). Finally, by a standard truncation and approximation argument, we extend the result to all $u \in W_{0}^{1, p}(\Omega)$ and then to the case when, possibly, $|\Omega|=\infty$.

Proof of Theorem B. As in the proof of Theorem A, we can restrict ourselves to the case when $u \in W_{0}^{1, p}(\Omega) \cap L^{\infty}(\Omega)$. We fix such $u$ and assume that $t_{k}$ and $a_{k}$ have the same meaning as in (2.3). Given $m \in \mathbb{N}$, we have (using $a_{1}=0$ )

$$
\begin{aligned}
0 & \leq \frac{a_{m}^{n}}{m^{n-1}}=\sum_{k=1}^{m-1}\left(\frac{a_{k+1}^{n}}{(k+1)^{n-1}}-\frac{a_{k}^{n}}{k^{n-1}}\right) \\
& =\sum_{k=1}^{m-1} \frac{a_{k+1}^{n}-a_{k}^{n}}{(k+1)^{n-1}}-\sum_{k=1}^{m-1} a_{k}^{n}\left(\frac{1}{k^{n-1}}-\frac{1}{(k+1)^{n-1}}\right) .
\end{aligned}
$$


Hence (passing to limit for $m \rightarrow \infty$ )

$$
\begin{aligned}
\sum_{k=1}^{\infty} \frac{a_{k}^{n}}{k^{n}} & \leq C \sum_{k=1}^{\infty} a_{k}^{n}\left(\frac{1}{k^{n-1}}-\frac{1}{(k+1)^{n-1}}\right) \leq C \sum_{k=1}^{\infty} \frac{a_{k+1}^{n}-a_{k}^{n}}{(k+1)^{n-1}} \\
& \leq C \sum_{k=1}^{\infty} \frac{a_{k+1}^{n-1}\left(a_{k+1}-a_{k}\right)}{(k+1)^{n-1}} \\
& \leq C\left(\sum_{k=1}^{\infty}\left(a_{k+1}-a_{k}\right)^{n}\right)^{\frac{1}{n}}\left(\sum_{k=1}^{\infty} \frac{a_{k+1}^{n}}{(k+1)^{n}}\right)^{1-\frac{1}{n}} .
\end{aligned}
$$

Recalling that $a_{1}=0$, we infer from (2.8) and (2.9) that

$$
\sum_{k=1}^{\infty} \frac{u^{*}\left(t_{k}\right)^{n}}{k^{n}}=\sum_{k=1}^{\infty} \frac{a_{k}^{n}}{k^{n}} \leq C \sum_{k=1}^{\infty}\left(a_{k+1}-a_{k}\right)^{n} \leq C \int_{\Omega}|\nabla u|^{n} d x,
$$

a discrete version of (1.7).

\section{The SPACE $W_{p}(\Omega)$}

Let us now have a closer look at the new function space $W_{p}(\Omega)$.

Theorem 3.1. Assume that $|\Omega|<\infty$ and $p \in[1, \infty)$. Then:

(i) $\left\|\chi_{E}\right\|_{W_{p}(\Omega)}=(\log 2)^{\frac{1}{p}}$ for every measurable $E \subset \Omega$;

(ii) $L^{\infty}(\Omega) \varsubsetneqq W_{p}(\Omega)$;

(iii) each integer-valued $u \in W_{p}(\Omega)$ is bounded;

(iv) $W_{p}(\Omega)$ is not a linear set;

(v) $W_{p}(\Omega) \varsubsetneqq B W_{p}(\Omega)$.

Proof. (i) For every $E \subset \Omega$, we have

$$
\left\|\chi_{E}\right\|_{W_{p}(\Omega)}=\left(\int_{|E|}^{2|E|} \frac{d t}{t}\right)^{\frac{1}{p}}=(\log 2)^{\frac{1}{p}} .
$$

(ii) First, let $\|u\|_{\infty} \leq 1$. Then

$$
\begin{aligned}
\int_{0}^{|\Omega|} & \frac{\left(u^{*}\left(\frac{t}{2}\right)-u^{*}(t)\right)^{p}}{t} d t \leq \int_{0}^{|\Omega|} \frac{u^{*}\left(\frac{t}{2}\right)-u^{*}(t)}{t} d t \\
& =\lim _{\varepsilon \rightarrow 0_{+}} \int_{\varepsilon}^{\frac{1}{\varepsilon}} \frac{u^{*}\left(\frac{t}{2}\right)-u^{*}(t)}{t} d t=\lim _{\varepsilon \rightarrow 0_{+}}\left(\int_{\varepsilon}^{\frac{1}{\varepsilon}} \frac{u^{*}\left(\frac{t}{2}\right)}{t} d t-\int_{\varepsilon}^{\frac{1}{\varepsilon}} \frac{u^{*}(t)}{t} d t\right) \\
& =\lim _{\varepsilon \rightarrow 0_{+}}\left(\int_{\frac{\varepsilon}{2}}^{\frac{1}{2 \varepsilon}} \frac{u^{*}(t)}{t} d t-\int_{\varepsilon}^{\frac{1}{\varepsilon}} \frac{u^{*}(t)}{t} d t\right) \leq \lim _{\varepsilon \rightarrow 0_{+}} \int_{\frac{\varepsilon}{2}}^{\varepsilon} \frac{u^{*}(t)}{t} d t=(\log 2)\|u\|_{\infty} .
\end{aligned}
$$

Since $W_{p}(\Omega)$ is obviously closed with respect to the multiplication by a scalar, we get $L^{\infty}(\Omega) \subset W_{p}(\Omega)$. We have to show that this inclusion is strict. To this end, let $u$ be any measurable function defined on $\Omega$ such that $u^{*}(t)=\left(\log \frac{|\Omega|}{t}\right)^{\alpha}, t \in\left(0, \frac{|\Omega|}{2}\right)$, with $\alpha \in(0,1)$ to be determined later. Then

$$
u^{*}\left(\frac{t}{2}\right)-u^{*}(t)=\int_{\frac{t}{2}}^{t} \alpha\left(\log \frac{|\Omega|}{s}\right)^{\alpha-1} \frac{d s}{s} \leq \alpha \log 2\left(\log \frac{|\Omega|}{t}\right)^{\alpha-1} .
$$


Thus, for $\alpha \in\left(0, \frac{p-1}{p}\right), u$ is unbounded, but

$$
\|u\|_{W_{p}(\Omega)}^{p} \leq C \int_{0}^{|\Omega|}\left(\log \frac{|\Omega|}{t}\right)^{p(\alpha-1)} \frac{d t}{t}<\infty .
$$

(iii) Suppose that $u$ is an integer-valued unbounded function on $\Omega$. Then there are $\alpha_{1}>\alpha_{2}>\cdots>0$ such that $u^{*}\left(\alpha_{j}-\right)-u^{*}\left(\alpha_{j}+\right) \geq 1, j \in \mathbb{N}$. For each $j=1,2, \ldots$, we have

$$
u^{*}\left(\frac{t}{2}\right)-u^{*}(t) \geq 1, \quad \alpha_{j} \leq t<2 \alpha_{j},
$$

and thus

$$
\int_{\alpha_{j}}^{2 \alpha_{j}} \frac{\left(u^{*}\left(\frac{t}{2}\right)-u^{*}(t)\right)^{p}}{t} d t \geq \int_{\alpha_{j}}^{2 \alpha_{j}} \frac{d t}{t}=\log 2 .
$$

From the system $\left\{\left(\alpha_{j}, 2 \alpha_{j}\right)\right\}$ of intervals we may obviously select an infinite disjoint subsystem, and thus

$$
\int_{0}^{|\Omega|} \frac{\left(u^{*}\left(\frac{t}{2}\right)-u^{*}(t)\right)^{p}}{t} d t=\infty
$$

whence $u \notin W_{p}(\Omega)$.

(iv) It follows from (ii) that there is a non-negative function $u \in W_{p}(\Omega)$ such that $u^{*}$ is unbounded. Let $[u]$ be the integer part of $u$ and set $w:=[u]+1, v:=w-u$. Then $|v| \leq 1$, thus $v \in W_{p}(\Omega)$ by (ii). On the other hand, $w=u+v$ is integer valued but unbounded, hence $w \notin W_{p}(\Omega)$ by (iii).

(v) The (discrete version of) the inclusion $W_{p}(\Omega) \subset B W_{p}(\Omega)$ was shown in the proof of Theorem B. The inclusion is strict indeed, since $B W_{p}(\Omega)$ is a linear set, whereas $W_{n}(\Omega)$ is not.

Note that we have in fact shown that

$$
W_{p}(\Omega)+L^{\infty}(\Omega) \not \subset W_{p}(\Omega) .
$$

\section{ACKNOWLEDGMent}

This research was partly supported by MSM 113200007, the grant of the Grant Agency of the Czech Republic no. 201/00/0767, and the grant of the Grant Agency of the Charles University no. 165/99. We would like to thank Piotr Hajłasz for fruitful discussions which turned our attention to modern methods of proving Sobolev inequalities.

\section{REFERENCES}

[1] S. Bakry, T. Coulhon, M. Ledoux, and L. Saloff-Coste, Sobolev inequalities in disguise, Indiana Univ. Math. J. 441995 1033-1074. MR 97c:46039

[2] C. Bennett, R. De Vore and R. Sharpley, Weak-L $L^{\infty}$ and BMO, Annals of Math. 1131981 601-611. MR 82h:46047

[3] C. Bennett and R. Sharpley, Interpolation of Operators, Pure and Applied Mathematics Vol. 129, Academic Press, Boston, 1988. MR 89e:46001

[4] H. Brézis and S. Wainger, A note on limiting cases of Sobolev embeddings and convolution inequalities, Comm. Partial Diff. Eq. 51980 773-789. MR 81k:46028

[5] A. Cianchi, A sharp embedding theorem for Orlicz-Sobolev spaces, Indiana Univ. Math. J. 451996 39-65. MR 97h:46044

[6] D. E. Edmunds, R. Kerman and L. Pick, Optimal Sobolev imbeddings involving rearrangement-invariant quasinorms, J. Funct. Anal. 1702000 307-355. MR 2000m:46070 
[7] H. Federer, Geometric Measure Theory, Springer, Berlin, 1969 (Second edition 1996). MR 41:1976

[8] K. Hansson, Imbedding theorems of Sobolev type in potential theory, Math. Scand. 45 1979, 77-102. MR 81j:31007

[9] J. A. Hempel, G. R. Morris and N. S. Trudinger, On the sharpness of a limiting case of the Sobolev imbedding theorem, Bull. Australian Math. Soc. 3 1970, 369-373. MR 43:6717

[10] J. Malý and W. P. Ziemer, Fine regularity of solutions of elliptic partial differential equations, AMS Mathematical Surveys and Monographs Vol. 51, Amer. Math. Soc., Providence, 1997. MR 98h:35080

[11] V. G. Maz'ya, A theorem on the multidimensional Schrödinger operator, (Russian), Izv. Akad. Nauk. 28 1964, 1145-1172.

[12] V. G. Maz'ya, Sobolev Spaces, Springer, Berlin, 1975.

[13] R. O'Neil, Convolution operators and $L_{(p, q)}$ spaces, Duke Math. J. 30 1963, 129-142. MR 26:4193

[14] J. Peetre, Espaces d'interpolation et théorème de Soboleff, Ann. Inst. Fourier 16 1966, 279317. MR 36:4334

[15] S. I. Pokhozhaev, On the eigenfunctions of the equation $\Delta u+\lambda f(u)=0$, Dokl. Akad. Nauk SSR 1651965 36-39. MR 33:411

[16] S. L. Sobolev, Applications of Functional Analysis in Mathematical Physics, Transl. of Mathem. Monographs, American Math. Soc. Vol. 7, Providence, 1963. MR 29:2624

[17] E. M. Stein, Singular Integrals and Differentiability Properties of Functions Princeton University Press, Princeton, NJ, 1970. MR 44:7280

[18] L. Tartar, Imbedding theorems of Sobolev spaces into Lorentz spaces, Boll. Un. Mat. Ital. 8 1-B 1998, 479-500. MR 99k:46060

[19] N.S. Trudinger, On imbeddings into Orlicz spaces and some applications, J. Math. Mech. 17 1967, 473-483. MR 35:7121

[20] V.I. Yudovich, Some estimates connected with integral operators and with solutions of elliptic equations, Soviet Math. Doklady 138 1961, 805-808. MR 25:4236]

Department of Mathematical Analysis, Faculty of Mathematics and Physics, Charles University, Sokolovská 83, 18675 Praha 8, Czech Republic

E-mail address: maly@karlin.mff.cuni.cz

Department of Mathematical Analysis, Faculty of Mathematics and Physics, Charles University, Sokolovská 83, 18675 Praha 8, Czech Republic

E-mail address: pick@karlin.mff.cuni.cz 